\title{
MONITORING RIVER PERIPHYTON WITH ARTIFICIAL BENTHIC SUBSTRATES ${ }^{1}$
}

\author{
Rex L. LOWE \& William F. GALE
}

Department of Biological Sciences, Bowling Green State University, Bowling Green, Ohio 43403; University of Michigan Biological Station, Pellston, Michigan 49769

1 This study was partially supported by the Pennsylvania Power and Light Company

${ }^{2}$ Ichthyological Associates, Inc., RD I, Berwick, Pennsylvania 18603

Received April 20, 1979

Keywords: periphyton, diatom, benthic, monitoring, algae

\begin{abstract}
The objective of this research was to identify the materials and methods necessary to study the attached algal community on a river bottom in deep water. The study site was the Susquehanna River near Falls, Pennsylvania. Artificial substrates of smooth glass, frosted glass, Vermont slate, 'sandy slate' (flagstone) and acrylic plate were placed on the stream bottom in detritus free sample holders by scuba divers. Both monthly and long-term cumulative samples were collected from the plates employing scuba and a Bar-Clamp sampler. River stones (natural substrates) were collected for comparison. Samples were analyzed in a Palmer Cell under a Bausch and Lomb research microscope. Diatoms were the most important colonizers of river stones, with the genera Nitzschia and Navicula most abundant. Highest periphyton densities occurred on natural substrates in winter with a maximum of $2.2 \times 10^{4}$ units $/ \mathrm{mm}^{2}$. Artificial substrates with one month exposure periods accumulated maximum periphyton density from May through October with relatively low densities in winter. Cumulative artificial substrates were most like river stones in patterns of colonization. Frosted acrylic is recommended for future studies employing benthic artificial periphyton substrates.
\end{abstract}

\section{Introduction}

Phycoperiphyton, periphytic algae, has been employed extensively in lotic water quality research (Lowe \& McCullough, 1974; Lowe, 1972; Butcher, 1947; Dam, 1974; VanLandingham, 1976; Patrick, 1968, 1973). In most instances, some sort of artificial substrate has been

Dr. W. Junk b.v. Publishers - The Hague, The Netherlands employed and the phycoperiphyton colonizing the substrate analyzed for community structure (Patrick et al., 1954; Patrick \& Hohn, 1956; Hohn \& Hellerman, I963; Lowe \& McCullough, 1974). Most periphyton samplers have been modeled after the Catherwood Diatometer of Patrick \& Hohn, 1956. This has become a popular and successful means of monitoring water quality (Patrick, 1973). The diatometer is normally placed in the stream channel and floats a few centimeters below the surface. The community of algae colonizing this substrate, although useful in monitoring the water quality, may be qualitatively and quantitatively different than the community colonizing the substrate on the stream bottom. This may be particularly true in deep, clear rivers with good light penetration. The effect of water quality on the periphyton communities as they exist in the stream may best be measured by observing communities collected directly from the stream bottom. The objective of this study was to identify a practical artificial substrate for studying phycoperiphyton on the bottom of a stream. Our criteria for the substrate required that it be inexpensive, readily available, easy to handle and support a community representative of the natural community.

\section{Methods and materials}

The site at which periphyton studies were conducted was the Susquehanna River near Falls, Pennsylvania. Water depth at the sampling site varied from 2.5 to $6 \mathrm{~m}$ during the investigation. Artificial substrates tested for peri- 
phyton colonization included (I) Vermont slate (roughened by sandblasting; (2) 'Sandy Slate' (flagstone); (3) smooth glass; (4) frosted glass and (5) frosted acrylic. All substrate plates were $14 \times 14 \mathrm{~cm}$ and were fastened by brass pins to an acrylic holder (Fig. I), which lacked projections that might catch drifting detritus. The plates faced upstream at $5^{\circ}$ from horizontal so that they offered little resistance to current and accumulated little silt or detritus. The holder and plates were lowered to the river bottom at mid-channel by a scuba diver on a submersible raft (Gale \& Thompson, 1974) and fastened into position with steel stakes driven into the substrate. The front of the holder and steel stakes were covered with small stones to prevent detritus from catching on them.

Sixteen plates of Vermont slate, with one-half of the upper surface of each covered by acrylic, were placed in the river late in 1973 . Three plates were randomly selected for monthly sampling at each station. The three slots where plates were removed were filled with clean plates for the following monthly samples. The remaining 13 plates provided samples of cumulative periphyton and were exposed from two to thirteen months. In April, 1974, plates of smooth and frosted glass and sandy slate were placed in a second holder on the river bottom beside the first one.

For comparison, samples were also collected from river stones in water with a minimum depth of $1.5 \mathrm{~m}$. Table $I$ indicates the schedule followed for sampling artificial substrates and river stones.

Samples were collected employing a Bar-Clamp sampler and scuba (Gale, 1975). Periphyton was removed from the substrate in the laboratory by vibration with an ultrasonic dental cleaning probe. After settling five or
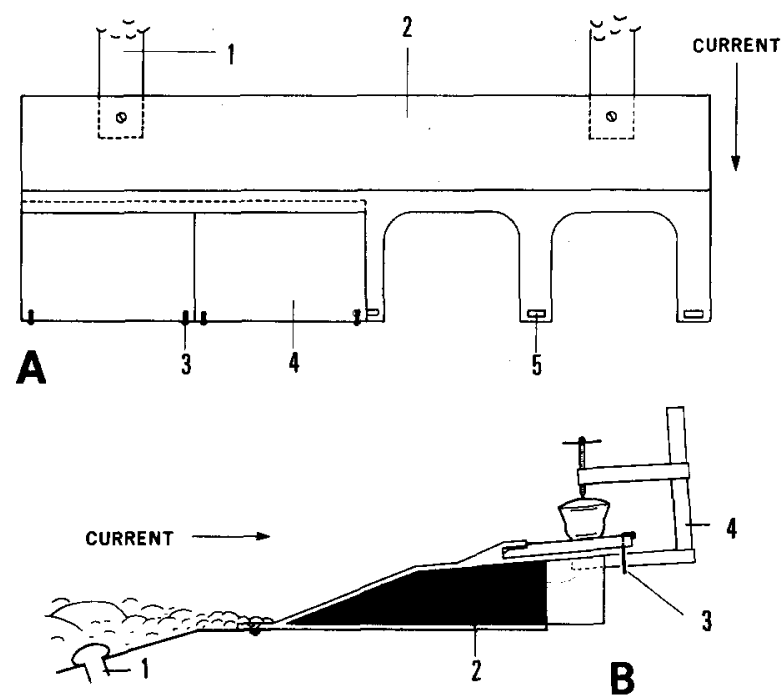

Fig. I. Detritus-free apparatus for periphytic algae studies: A. Acrylic holder with two plates removed (top view): I) metal retaining strap; 2) deflecting shield, acrylic;3) brass pin; 4) acrylic plate; 5) pin retaining slot. B. Acrylic holder (end view) with sampler in place: 1) steel stake (buried); 2) concrete ballast; 3) brass pin; 4) bar-clamp sampler.

more days, periphyton samples were concentrated to 50 $\mathrm{ml}$ by decanting and analyzed and enumerated in the laboratory employing a Palmer-Malony counting chamber and a Bausch and Lomb research microscope operating at a total magnification of $430 \mathrm{X}$. In most instances, 750 units (Gale \& Lowe, I97I) were enumerated and identified to genus in each sample (about 250/each of 3 subsamples). Extremely low algal densities in some samples made it impractical to count 750 units. The most

Table 1. Sampling schedule for periphyton on artificial substrates and river stones.

Substrates

Dates Sampled (months)

\begin{tabular}{|c|c|c|c|c|c|c|c|c|c|c|c|c|c|}
\hline & $\mathbf{J}$ & $\mathbf{F}$ & $\mathbf{M}$ & A & $\mathbf{M}$ & $\mathbf{J}$ & $\mathrm{J}$ & $\mathbf{A}$ & $\mathbf{S}$ & $\mathrm{O}$ & $\mathbf{N}$ & D & $\mathrm{J}$ \\
\hline Vermont Slate & $\mathrm{x}$ & $x$ & $\mathbf{x}$ & $\mathbf{x}$ & $\mathbf{x}$ & $\mathbf{x}$ & $\mathbf{x}$ & $\mathbf{x}$ & $\mathbf{x}$ & $\mathrm{x}$ & $\mathbf{x}$ & $\mathrm{x}$ & $\mathbf{x}$ \\
\hline Sandy Slate & & & & & & $\mathbf{x}$ & $\mathrm{x}$ & $\mathrm{x}$ & $\mathbf{x}$ & $\mathbf{x}$ & $\mathbf{x}$ & & $\mathbf{x}$ \\
\hline Frosted Acrylic & $\mathbf{x}$ & $\mathrm{x}$ & $\mathbf{x}$ & $\mathrm{x}$ & $\mathbf{x}$ & $\mathbf{x}$ & $\mathrm{x}$ & $\mathbf{x}$ & $\mathbf{x}$ & $\mathrm{x}$ & $\mathrm{x}$ & $\mathrm{x}$ & $\mathrm{x}$ \\
\hline Smooth Glass & & & & & $\mathrm{x}$ & $\mathrm{x}$ & $\mathrm{x}$ & $\mathrm{x}$ & $\mathbf{x}$ & $\mathbf{x}$ & $\mathbf{x}$ & $\mathbf{x}$ & $\mathbf{x}$ \\
\hline Frosted Glass & & & & & $\mathbf{x}$ & $\mathbf{x}$ & $\mathbf{x}$ & $\mathbf{x}$ & $\mathrm{x}$ & $\mathbf{x}$ & $\mathrm{x}$ & $\mathbf{x}$ & $\mathbf{x}$ \\
\hline Cumulative Vermont Slate & & & $\mathbf{x}$ & $\mathbf{x}$ & $\mathbf{x}$ & $\mathbf{x}$ & $\mathrm{x}$ & $\mathbf{x}$ & $\mathbf{x}$ & $\mathbf{x}$ & $\mathbf{x}$ & $\mathbf{x}$ & $\mathbf{x}$ \\
\hline Cumulative Frosted Acrylic & & & & & $\mathbf{x}$ & & $\mathrm{x}$ & & $\mathbf{x}$ & & $\mathbf{x}$ & $\mathrm{x}$ & \\
\hline River Stones (Shore) & $\mathrm{x}$ & $\mathrm{x}$ & & & $\mathrm{x}$ & & $\mathrm{x}$ & & $\mathbf{x}$ & & $\mathrm{x}$ & $\mathrm{x}$ & \\
\hline River Stones (Channel) & & & & & & & & & & & & $\mathbf{x}$ & $\mathbf{x}$ \\
\hline
\end{tabular}


abundant taxa in each sample were identified to species. Species identification often necessitated a magnification of $1000 \mathrm{X}$ with an oil immersion objective. Soft forms were identified in wet mounts and diatoms were identified from Hyrax mounts.

\section{Results}

A total of 38 genera of algae was identified in samples from natural river stones. Diatoms were the most important group of algae with the genera Nitzschia and Navicula most often dominating the community (Table 2). The stones supported $3.8 \times 10^{3}$ units of periphyton $/ \mathrm{mm}^{2}$ in January (Fig. 2). Nitzschia dissipata (Kütz.) Grun. was the most abundant species. In February the periphyton standing crop reached $2.2 \times 10^{4}$ units $/ \mathrm{mm}^{2}$ as $N$. dissipata continued to dominate the community. The May, July and September collections all yielded less than $2 \times 10^{3}$ units $/ \mathrm{mm}^{2}$. The population of $N$. dissipata had decreased both relatively and absolutely in these samples. Taxa becoming relatively more abundant during these months included Cocconeis placentula Ehr., Navicula cryptocephala Kütz., N. cryptocephala var. veneta(Kütz.) Rabh., $N$. viridula, Kütz. N. tripunctata (O. F. Müll.) Bory, $N$. salinarum var. intermedia (Grun.) $\mathrm{Cl}$., Cyclotella pseudostelligera Hust., Ankistrodesmus convolutus Corda and Scenedesmus quadracauda (Terp.) Breb., the latter three probably representing plankton fallout. In November and December, standing crops were again around $4 \times 10^{3}$ units $/ \mathrm{mm}^{2}$ and increased to $7.3 \times 10^{4}$ units $/ \mathrm{mm}^{2}$ in January, 1975. Diatoms were responsible for much of the fall-winter increase, the most important species being Cocconeis placentula, Cyclotella pseudostelligera, Navicula cryptocephala var. veneta, $N$. salinarum var. intermedia, N. tripunctata and Nitzschia dissipata. The com-

Table 2. Changes in number of genera of algae colonizing monthly artificial substrates at Falls, 1974-5.

\begin{tabular}{llc}
\hline Month & Substrate & No. Genera Present \\
\hline 1974 & & \\
Jan & Acrylic & 11 \\
& Vermont slate & 0 \\
Feb & Acrylic & - \\
& Vermont slate & 4 \\
Mar & Acrylic & 16 \\
& Vermont slate & 5
\end{tabular}

continued

\begin{tabular}{|c|c|c|}
\hline Month & Substrate & No. Genera Present \\
\hline \multirow[t]{2}{*}{ Apr } & Acrylic & 18 \\
\hline & Vermont slate & 4 \\
\hline \multirow[t]{5}{*}{ May } & Acrylic & 12 \\
\hline & Vermont slate & 9 \\
\hline & Smooth glass & 18 \\
\hline & Frosted glass & 17 \\
\hline & 'Sandy' slate & - \\
\hline \multirow[t]{5}{*}{ Jun } & Acrylic & 27 \\
\hline & Vermont slate & 19 \\
\hline & Smooth glass & 22 \\
\hline & Frosted glass & 19 \\
\hline & 'Sandy' slate & 24 \\
\hline \multirow[t]{5}{*}{ Jul } & Acrylic & 30 \\
\hline & Vermont slate & 25 \\
\hline & Smooth glass & 21 \\
\hline & Frosted glass & 28 \\
\hline & 'Sandy' slate & 21 \\
\hline \multirow[t]{5}{*}{ Aug } & Acrylic & 27 \\
\hline & Vermont slate & 24 \\
\hline & Smooth glass & 30 \\
\hline & Frosted glass & 30 \\
\hline & 'Sandy' slate & 23 \\
\hline \multirow[t]{5}{*}{ Sep } & Acrylic & 20 \\
\hline & Vermont slate & 18 \\
\hline & Smooth glass & 23 \\
\hline & Frosted glass & 23 \\
\hline & 'Sandy' slate & 25 \\
\hline \multirow[t]{5}{*}{ Oct } & Acrylic & 21 \\
\hline & Vermont slate & 20 \\
\hline & Smooth glass & 18 \\
\hline & Frosted glass & 17 \\
\hline & 'Sandy' slate & 19 \\
\hline \multirow[t]{5}{*}{ Nov } & Acrylic & 11 \\
\hline & Vermont slate & 6 \\
\hline & Smooth glass & 8 \\
\hline & Frosted glass & 16 \\
\hline & 'Sandy' slate & 13 \\
\hline \multirow[t]{5}{*}{ Dec } & Acrylic & 8 \\
\hline & Vermont slate & 3 \\
\hline & Smooth glass & - \\
\hline & Frosted glass & 6 \\
\hline & 'Sandy' slate & - \\
\hline \multicolumn{3}{|l|}{1975} \\
\hline \multirow[t]{5}{*}{ Jan } & Acrylic & 5 \\
\hline & Vermont slate & 7 \\
\hline & Smooth glass & 9 \\
\hline & Frosted glass & 7 \\
\hline & 'Sandy' slate & 7 \\
\hline
\end{tabular}




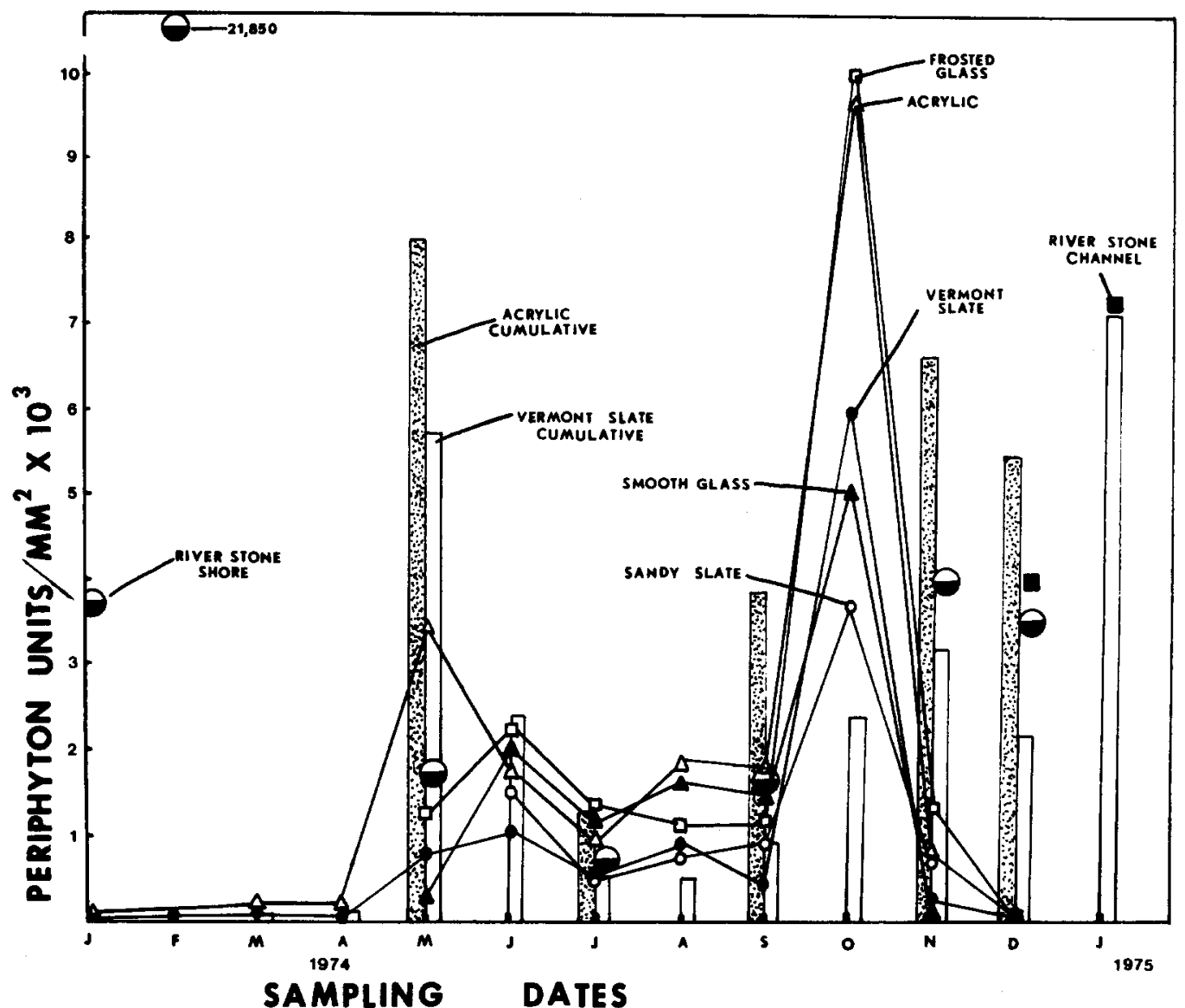

Fig. 2. Density of periphyton on various substrates submerged in the Susquehanna River from January, 1974 to January, 1975 .

munity dynamics of phycoperiphyton on river stones can be summarized as follows: relatively large standing crops in January and February, decreasing to a minimum in mid-summer, then increasing to a winter maximum again.

All of the substrates which were allowed one month colonization periods (frosted acrylic, Vermont slate, sandy slate, frosted glass and smooth glass) displayed community dynamics similar to one another (Fig. 2). Colonization rates were very low from January through April on both Vermont slate and acrylic substrates. The largest standing crop observed during this period was I. $4 \times 10^{2}$ units $/ \mathrm{mm}^{2}$ on frosted acrylic in March. Most of the colonizers were diatoms of the genera Navicula and Nitzschia (Table 3). Frosted acrylic and Vermont slate were more heavily colonized in May with $3.4 \times \mathrm{Xo}^{3}$ and $7.7 \times \mathrm{IO}^{2}$ units of phycoperiphyton $/ \mathrm{mm}^{2}$. Expanding populations of Nitzschia dissipata, N. palea (Kütz.) W. Sm., Navicula cryptocephala, $N$. cryptocephala var. veneta, $N$. tripunctata, $N$. viridula and Cymbella minuta Hilse ex Rabh. accounted for most of the increase in community density. Smooth and frosted glass substrates were collected for the first time in May and supported $2.4 \times 10^{2}$ and $\mathrm{I} .2 \times 10^{3}$ units $/ \mathrm{mm}^{2}$ respectively. In June, sandy slate was collected for the first time. All five artificial substrates collected in June supported standing crops between $1.1 \times 10^{3}$ and $2.3 \times 10^{3}$ units $/ \mathrm{mm}^{2}$. All substrates displayed relatively stable colonization rates until October when colonization rates on all artificial substrates increased by at least 3 fold. Vermont slate displayed the greatest increase in algal density when the standing crop increased from $3.6 \times 10^{2}$ units $/ \mathrm{mm}^{2}$ in September to $5.9 \times 10^{3}$ units $/ \mathrm{mm}^{2}$ in October. Frosted glass supported the densest community of the October samples $\left(1.0 \times 10^{4}\right.$ 
Table 3. Most abundant three genera of algae on River stones and artificial substrates $(M=$ monthly; $C=$ cumulative $)$ at Falls 1974-5. Genera with identical values are listed alphabetically if all can be included; otherwise, none are included.

Month

\begin{tabular}{|c|c|c|c|}
\hline & Substrate & Genera & $\%$ Total \\
\hline \multicolumn{4}{|c|}{1974} \\
\hline \multirow[t]{7}{*}{ Jan } & \multirow{3}{*}{$\begin{array}{c}\text { River stone } \\
\text { (shore) }\end{array}$} & Nitzschia & 51.3 \\
\hline & & Navicula & 24.8 \\
\hline & & Cocconeis & 7.0 \\
\hline & \multirow[t]{3}{*}{ Acrylic (M) } & Nitzschia & 59.3 \\
\hline & & Navicula & 20.0 \\
\hline & & Oscillatoria & 7.4 \\
\hline & \multicolumn{2}{|l|}{ Vermont slate $(\mathrm{M})$} & 0 \\
\hline \multirow[t]{5}{*}{ Feb } & \multirow{3}{*}{$\begin{array}{l}\text { River stone } \\
\text { (shore) }\end{array}$} & Nitzschia & 73.4 \\
\hline & & Navicula & 11.1 \\
\hline & & Synedra & 7.5 \\
\hline & \multicolumn{2}{|l|}{ Acrylic (M) } & -- \\
\hline & Vermont slate $(M)$ & Nitzschia & 57.0 \\
\hline \multirow[t]{8}{*}{ Mar } & \multirow[t]{2}{*}{ Acrylic (M) } & Nitzschia & 59.3 \\
\hline & & Navicula & 18.5 \\
\hline & \multicolumn{2}{|l|}{ Acrylic (C) } & - \\
\hline & \multirow[t]{3}{*}{ Vermont slate (M) } & Navicula & 32.9 \\
\hline & & Nitzschia & 24.2 \\
\hline & & Asterionella & 21.9 \\
\hline & \multirow[t]{2}{*}{ Vermont slate (C) } & Navicula & 40.6 \\
\hline & & Nitzschia & 40.6 \\
\hline \multirow[t]{8}{*}{ Apr } & \multirow[t]{3}{*}{ Acrylic (M) } & Navicula & 49.6 \\
\hline & & Gomphonema & 17.6 \\
\hline & & Meridion & 6.9 \\
\hline & \multirow[t]{2}{*}{ Vermont slate $(\mathrm{M})$} & Navicula & 42.9 \\
\hline & & Cymbella & 28.6 \\
\hline & \multirow[t]{3}{*}{ Vermont slate (C) } & Fragilaria & 33.3 \\
\hline & & Navicula & 20.8 \\
\hline & & Nitzschia & 12.5 \\
\hline \multirow[t]{9}{*}{ May } & \multirow{3}{*}{$\begin{array}{l}\text { River stone } \\
\text { (shore) }\end{array}$} & Navicula & 55.9 \\
\hline & & Nitzschia & 18.5 \\
\hline & & Cocconeis & 11.0 \\
\hline & \multirow[t]{3}{*}{ Acrylic (M) } & Navicula & 47.8 \\
\hline & & Nitzschia & 36.9 \\
\hline & & Cymbella & 4.6 \\
\hline & \multirow[t]{3}{*}{ Acrylic (C) } & Nitzschia & 50.8 \\
\hline & & Navicula & 33.5 \\
\hline & & Gomphonema & 7.5 \\
\hline
\end{tabular}

Table 3. Continued

\begin{tabular}{|c|c|c|c|}
\hline \multicolumn{4}{|c|}{ Month } \\
\hline & Substrate & Genera & \% Total \\
\hline & \multirow[t]{3}{*}{ Vermont slate $(\mathbf{M})$} & Navicula & 41.6 \\
\hline & & Nitzschia & 38.9 \\
\hline & & Cymbella & 7.4 \\
\hline & \multirow[t]{3}{*}{ Vermont slate (C) } & Nitzschia & 71.3 \\
\hline & & Navicula & 25.6 \\
\hline & & Gomphonema & 1.1 \\
\hline & \multirow[t]{3}{*}{ Smooth glass } & Navicula & 33.6 \\
\hline & & Nitzschia & 19.3 \\
\hline & & Cymbella & 15.1 \\
\hline & \multirow[t]{3}{*}{ Frosted glass } & Navicula & 52.6 \\
\hline & & Nitzschia & 24.0 \\
\hline & & Cymbella & 11.8 \\
\hline & \multicolumn{2}{|l|}{ 'Sandy' slate } & 0 \\
\hline \multirow[t]{18}{*}{ Jun } & \multirow[t]{3}{*}{ Acrylic (M) } & Navicula & 24.2 \\
\hline & & Nitzschia & 20.6 \\
\hline & & Ankistrodesmus & 14.0 \\
\hline & \multirow[t]{3}{*}{ Vermont slate (M) } & Navicula & 43.4 \\
\hline & & Nitzschia & 13.8 \\
\hline & & Ankistrodesmus & 12.7 \\
\hline & \multirow[t]{3}{*}{ Vermont slate (C) } & Nitzschia & 54.6 \\
\hline & & Navicula & 26.2 \\
\hline & & Stephanodiscus & 5.8 \\
\hline & \multirow[t]{3}{*}{ Smooth glass } & Cyclotella & 21.8 \\
\hline & & Navicula & 19.8 \\
\hline & & Nitzschia & 12.5 \\
\hline & \multirow[t]{3}{*}{ Frosted glass } & Navicula & 21.2 \\
\hline & & Cyclotella & 19.4 \\
\hline & & Nitzschia & 18.8 \\
\hline & \multirow[t]{3}{*}{ 'Sandy' slate } & Navicula & 37.4 \\
\hline & & Nitzschia & 18.9 \\
\hline & & Stephanodiscus & 9.7 \\
\hline \multirow[t]{12}{*}{ Jul } & \multirow{3}{*}{$\begin{array}{l}\text { River stone } \\
\text { (shore) }\end{array}$} & Navicula & 23.9 \\
\hline & & Cocconeis & 23.1 \\
\hline & & Cyclotella & 18.8 \\
\hline & \multirow[t]{3}{*}{ Acrylic (M) } & Cyclotella & 27.6 \\
\hline & & Ankistrodesmus & 14.1 \\
\hline & & Stephanodiscus & 9.2 \\
\hline & \multirow{3}{*}{ Acrylic (C) } & Ankistrodesmus & 19.6 \\
\hline & & Navicula & 19.5 \\
\hline & & Cyclotella & 17.5 \\
\hline & \multirow[t]{3}{*}{ Vermont slate (M) } & Cyclotella & 23.2 \\
\hline & & Navicula & 13.0 \\
\hline & & Stephanodiscus & 11.8 \\
\hline
\end{tabular}


Table 3. Continued

\begin{tabular}{|c|c|c|c|}
\hline \multicolumn{4}{|l|}{ Month } \\
\hline & Substrate & Genera & $\%$ Total \\
\hline & \multirow[t]{3}{*}{ Vermont siate (C) } & Cyclotella & 20.7 \\
\hline & & Ankistrodesmus & 17.0 \\
\hline & & Scenedesmus & 11.4 \\
\hline & \multirow[t]{3}{*}{ Smooth glass } & Cyclotella & 30.3 \\
\hline & & Stephanodiscus & 13.5 \\
\hline & & Navicula & 10.8 \\
\hline & \multirow[t]{3}{*}{ Frosted glass } & Cyclotella & 24.3 \\
\hline & & Stephanodiscus & 11.6 \\
\hline & & Navicula & 9.5 \\
\hline \multirow[t]{3}{*}{. } & \multirow[t]{3}{*}{ 'Sandy' slate } & Navicula & 24.8 \\
\hline & & Cyclotella & 21.0 \\
\hline & & Cocconeis & 10.7 \\
\hline \multirow[t]{18}{*}{ Aug } & \multirow[t]{3}{*}{ Acrylic (M) } & Cyclotella & 25.3 \\
\hline & & Scenedesmus & 19.4 \\
\hline & & Stephanodiscus & 12.8 \\
\hline & \multirow[t]{3}{*}{ Vermont slate (M) } & Cyclotella & 24.3 \\
\hline & & Scenedesmus & 19.5 \\
\hline & & Stephanodiscus & 13.9 \\
\hline & \multirow[t]{3}{*}{ Vermont slate $(C)$} & Scenedesmus & 26.4 \\
\hline & & Cyclotella & 17.4 \\
\hline & & Dictyosphaerium & 12.8 \\
\hline & \multirow[t]{3}{*}{ Smooth glass } & Scenedesmus & 23.3 \\
\hline & & Cyclotella & 22.2 \\
\hline & & Stephanodiscus & 12.7 \\
\hline & \multirow[t]{3}{*}{ Frosted glass } & Cyclotella & 23.4 \\
\hline & & Scenedesmus & 17.4 \\
\hline & & Stephanodiscus & 17.4 \\
\hline & \multirow[t]{3}{*}{ 'Sandy' slate } & Cyclotella & 25.9 \\
\hline & & Scenedesmus & 17.5 \\
\hline & & Stephanodiscus & 12.0 \\
\hline \multirow[t]{14}{*}{ Sep } & \multirow{3}{*}{$\begin{array}{l}\text { River stone } \\
\text { (shore) }\end{array}$} & Cyclotella & 34.8 \\
\hline & & Navicula & 29.3 \\
\hline & & Cocconeis & 7.7 \\
\hline & \multirow[t]{2}{*}{ Acrylic (M) } & Cyclotella & 38.9 \\
\hline & & Navicula & 14.2 \\
\hline & \multirow[t]{3}{*}{ Acrylic (C) } & Cyclotella & 29.6 \\
\hline & & Stephanodiscus & 23.6 \\
\hline & & Navicula & 17.0 \\
\hline & \multirow[t]{3}{*}{ Vermont slate (M) } & Cyclotella & 41.4 \\
\hline & & Navicula & 24.2 \\
\hline & & Stephanodiscus & 9.9 \\
\hline & \multirow[t]{3}{*}{ Vermont slate (C) } & Cyclotella & 34.0 \\
\hline & & Navicula & 25.8 \\
\hline & & Stephanodiscus & 9.8 \\
\hline
\end{tabular}

Table 3.

\begin{tabular}{|c|c|c|c|}
\hline \multirow[t]{2}{*}{ Month } & \multirow[b]{2}{*}{ Substrate } & \multirow[b]{2}{*}{ Genera } & \multirow[b]{2}{*}{$\%$ Total } \\
\hline & & & \\
\hline & \multirow[t]{3}{*}{ Smooth glass } & Cyclotella & 30.0 \\
\hline & & Stephanodiscus & 24.1 \\
\hline & & Navicula & 14.7 \\
\hline & \multirow[t]{3}{*}{ Frosted glass } & Cyclotella & 42.3 \\
\hline & & Stephanodiscus & 12.2 \\
\hline & & Navicula & 12.0 \\
\hline & \multirow[t]{3}{*}{ 'Sandy' slate } & Cyclotella & 34.9 \\
\hline & & Navicula & 19.7 \\
\hline & & Stephanodiscus & 18.9 \\
\hline \multirow[t]{18}{*}{ Oct } & \multirow[t]{3}{*}{ Acrylic (M) } & Navicula & 44.9 \\
\hline & & Nitzschia & 26.1 \\
\hline & & Cyclotella & 8.3 \\
\hline & \multirow[t]{3}{*}{ Vermont slate (M) } & Navicula & 35.7 \\
\hline & & Nitzschia & 35.4 \\
\hline & & Cyclotella & 4.5 \\
\hline & \multirow[t]{3}{*}{ Vermont slate (C) } & Navicula & 47.5 \\
\hline & & Nitzschia & 19.9 \\
\hline & & Stephanodiscus & 8.3 \\
\hline & \multirow[t]{3}{*}{ Smooth glass } & Navicula & 36.7 \\
\hline & & Melosira & 17.6 \\
\hline & & Nitzschia & 14.4 \\
\hline & \multirow[t]{3}{*}{ Frosted glass } & Nitzschia & 29.3 \\
\hline & & Navicula & 28.0 \\
\hline & & Melósira & 11.0 \\
\hline & \multirow[t]{3}{*}{ 'Sandy' slate } & Navicula & 29.2 \\
\hline & & Cymbella & 27.5 \\
\hline & & Nitzschia & 20.5 \\
\hline \multirow[t]{15}{*}{ Nov } & \multirow{3}{*}{$\begin{array}{l}\text { River stone } \\
\text { (shore) }\end{array}$} & Navicula & 56.1 \\
\hline & & Nitzschia & 28.9 \\
\hline & & Cocconeis & 2.9 \\
\hline & \multirow[t]{3}{*}{ Acrylic (M) } & Navicula & 47.5 \\
\hline & & Nitzschia & 32.8 \\
\hline & & Phormidium & 8.2 \\
\hline & \multirow[t]{3}{*}{ Acrylic (C) } & Navicula & 38.2 \\
\hline & & Nitzschia & 32.7 \\
\hline & & Cocconeis & 6.0 \\
\hline & \multirow[t]{3}{*}{ Vermont slate (M) } & Navicula & 64.8 \\
\hline & & Nitzschia & 27.3 \\
\hline & & Fragilaria & 2.8 \\
\hline & \multirow[t]{3}{*}{ Vermont slate (C) } & Navicula & 53.0 \\
\hline & & Nitzschia & 26.2 \\
\hline & & Cocconeis & 5.9 \\
\hline
\end{tabular}


Table 3.

\begin{tabular}{|c|c|c|c|}
\hline \multirow[t]{2}{*}{ Month } & \multirow[b]{2}{*}{ Substrate } & \multirow[b]{2}{*}{ Genera } & \multirow[b]{2}{*}{$\%$ Total } \\
\hline & & & \\
\hline & \multirow[t]{3}{*}{ Smooth glass } & Navicula & 55.6 \\
\hline & & Nitzschia & 27.0 \\
\hline & & Cocconeis & 6.4 \\
\hline & \multirow[t]{2}{*}{ Frosted glass } & Navicula & 57.8 \\
\hline & & Nitzschia & 26.6 \\
\hline & \multirow[t]{3}{*}{ 'Sandy' slate } & Navicula & 60.5 \\
\hline & & Nitzschia & 29.5 \\
\hline & & Cymbella & 3.8 \\
\hline \multirow[t]{22}{*}{ Dec } & \multirow{3}{*}{$\begin{array}{l}\text { River stone } \\
\text { (shore) }\end{array}$} & Nitzschia & 49.3 \\
\hline & & Navicula & 35.6 \\
\hline & & Cymbella & 3.5 \\
\hline & \multirow{3}{*}{$\begin{array}{r}\text { River stone } \\
\text { (channel) }\end{array}$} & Nitzschia & 49.7 \\
\hline & & Navicula & 36.3 \\
\hline & & Cymbella & 2.0 \\
\hline & \multirow[t]{2}{*}{ Acrylic (M) } & Navicula & 43.9 \\
\hline & & Nitzschia & 12.2 \\
\hline & \multirow[t]{3}{*}{ Acrylic (C) } & Nitzschia & 39.7 \\
\hline & & Navicula & 35.0 \\
\hline & & Cocconeis & 6.6 \\
\hline & \multirow[t]{3}{*}{ Vermont slate (M) } & Navicula & 75.0 \\
\hline & & Gomphonema & 12.5 \\
\hline & & Nitzschia & 12.5 \\
\hline & \multirow[t]{3}{*}{ Vermont slate (C) } & Navicula & 46.0 \\
\hline & & Nitzschia & 44.8 \\
\hline & & Cocconeis & 3.6 \\
\hline & \multicolumn{2}{|l|}{ Smooth glass } & -- \\
\hline & \multirow[t]{3}{*}{ Frosted glass } & Nitzschia & 32.6 \\
\hline & & Diatoma & 21.7 \\
\hline & & Navicula & 21.7 \\
\hline & \multicolumn{2}{|l|}{ 'Sandy' slate } & -- \\
\hline \multirow{11}{*}{$\begin{array}{l}1975 \\
\text { Jan }\end{array}$} & & & \\
\hline & \multirow{3}{*}{$\begin{array}{r}\text { River stone } \\
\text { (channel) }\end{array}$} & Navicula & 50.9 \\
\hline & & Nitzschia & 42.8 \\
\hline & & Cymbella & 2.7 \\
\hline & \multirow[t]{2}{*}{ Acrylic (M) } & Navicula & 47.8 \\
\hline & & Cymbella & 21.7 \\
\hline & \multirow[t]{3}{*}{ Acrylic (C) } & Nitzschia & 58.7 \\
\hline & & Navicula & 24.3 \\
\hline & & Amphora & 9.6 \\
\hline & \multirow[t]{2}{*}{ Vermont slate (M) } & Melosira & 32.0 \\
\hline & & Nitzschia & 32.0 \\
\hline
\end{tabular}

Table 3. Continued

\begin{tabular}{llr}
\hline Month & & \\
\cline { 2 - 3 } Substrate & Genera & \% Total \\
\hline \multirow{2}{*}{ Vermont slate (C) } & Navicula & 50.7 \\
& Nitzschia & 43.8 \\
& Cocconeis & 2.2 \\
Smooth glass & Melosira & 33.3 \\
& Nitzschia & 21.6 \\
& Navicula & 19.6 \\
\multirow{2}{*}{ Frosted glass } & Melosira & 32.1 \\
& Navicula & 32.1 \\
& Nitzschia & 14.3 \\
& Navicula & 38.6 \\
'Sandy' slate & Gomphonema & 33.0 \\
& Nitzschia & 19.3 \\
\hline
\end{tabular}

units $/ \mathrm{mm}^{2}$ ). The increase in phycoperiphyton on all substrates in October was due primarily to expanding populations of Cymbella prostrata (Berk.) Cl., Melosira varians Ag., Navicula cryptocephala var. veneta, N. symmetrica Patr., Nitzschia dissipata and N. palea. Following the October standing crop maximum, all substrates supported relatively sparse communities in November with decreasing standing crops in December and January.

Cumulative diatom communities were collected from frosted acrylic and Vermont slate substrates that were exposed for longer periods than the monthly samples. It was felt that longer exposure periods might better simulate the community dynamics of phycoperiphyton on river stones. Frosted acrylic substrates were exposed from five to thirteen months with the 5 month sample collected in May, 1974 and the 13 month sample collected in January, 1975. The May collection from cumulative frosted acrylic yielded $1.0 \times 10^{4}$ units $/ \mathrm{mm}^{2}$ (Fig. 2). The substrate collected in July was exposed for 7 months and supported $1.3 \times 10^{3}$ units $/ \mathrm{mm}^{2}$. Following this minimum the phycoperiphyton increased in density in the September, November, December and January samples. The latter supporting $1.8 \times 10^{4}$ units $/ \mathrm{mm}^{2}$. Most of the increased density was due to increases in several species of Navicula and Nitzschia.

Cumulative Vermont slate substrates were exposed from three to thirteen months with the three month sample collected in March and the thirteen month sample collected in January. March and April collections each yielded less than 30 units of phycoperiphyton $/ \mathrm{mm}^{2}$ 
but in May $5.8 \times 10^{3}$ units $/ \mathrm{mm}^{2}$ were present (Fig. 2). $\mathrm{Na}$ vicula and Nitzschia composed over $95 \%$ of the May community. The most abundant species were Navicula cryptocephala, $N$. cryptocephala var. veneta, N. tripunctata, $N$. viridula, Nitzschia dissipata and $N$. palea. The standing crop fell to $2.4 \times 10^{3}$ units $/ \mathrm{mm}^{2}$ in June and to less than $10^{3}$ units $/ \mathrm{mm}^{2}$ in July, August and September. The most abundant genera in the summer months included Cyclotella, Scenedesmus, Ankistrodesmus and Dictyosphaerium. The standing crop increased to $2.3 \mathrm{x}$ $\mathrm{IO}^{3}$ units $/ \mathrm{mm}^{2}$ in October and by January had increased to $7.2 \times 10^{3}$ units $/ \mathrm{mm}^{2}$.

The structure of communities on various combinations of substrates was evaluated by determining the coefficient of community for pairs of substrates of different types and by contrasting pairs of substrates of different types in percent similarity tests (Whittaker \& Fairbanks, 1958). Coefficient of community indicates the percentage of genera that are shared by two samples and is determined by the formula $c c=\frac{c}{a+b-c}$, where $a$ is the number of genera in sample $I, b$ is the number of genera in sample 2 and $c$ is the number of genera in both samples. Percent similarity is determined by the formula PSc $=100-.5 \Sigma_{1 a}-b I$, where $\mathrm{a}$ and $\mathrm{b}$ are, for a genus, the percentage of samples $I$ and 2 which that genus represents. Percent similarity measures relative similarity of numerical composition and generally leads to grouping of communities by dominants (Whittaker \& Fairbanks, 1958).

In table 4 , communities colonizing the various substrates are compared. Replicate plates of Vermont slate usually displayed a relatively high percent similarity which exceeded $80 \%$ in five of eight months. When monthly Vermont slates (replicate I) for the period from May through December are combined and compared to combined slates (replicate 2 ), an overall percent similarity of 85 was obtained. Rather similar values were obtained for other substrates combined for the same period (Table 4).

In July, the standing crops on artificial substrates and river stones were fairly similar numerically, and it was thought that the communities various substrates and on river stones might have a high percent similarity

Table 4. Comparison of algal communities on artificial substrates $(M=$ monthly; $\mathrm{C}=$ cumulative) and River stones at Falls North Branch of the Susquehanna River as expressed by percent similarity and coefficient of community.

\begin{tabular}{|c|c|c|c|}
\hline Month & Substrates Compared & $\%$ Similarity & $\begin{array}{l}\text { Coefficient of } \\
\text { Community }\end{array}$ \\
\hline May & (M) Vermont slate (1) vs. (2) & 88 & 62 \\
\hline Jun & $"$ & 81 & 63 \\
\hline Jul & ", & 69 & 54 \\
\hline Aug & $"$ & 83 & 57 \\
\hline Sep & $"$ & 57 & 26 \\
\hline Oct & $"$ & 87 & 61 \\
\hline Nov & $"$ & 87 & 40. \\
\hline Dec & $"$ & 42 & 33 \\
\hline May-Dec & Acrylic vs. Vermont slate & 90 & 70 \\
\hline$"$ & Acrylis vs. Frosted glass & 86 & 75 \\
\hline " & Smooth glass vs. Frosted glass & 83 & 78 \\
\hline ", & Frosted glass vs. Vermont slate & 81 & 71 \\
\hline$"$ & Acrylic vs. Smooth Glass & 74 & 80 \\
\hline$"$ & Smooth glass vs. Vermont slate & 70 & 74 \\
\hline ", & Vermont slate (1) vs. (2) & 85 & 63 \\
\hline Jul & (M) Vermont slate vs. River stone & 78 & 52 \\
\hline$"$ & (M) 'Sandy' slate vs. River stone & 78 & 68 \\
\hline ", & (C) Acrylic vs. River stone & 72 & 45 \\
\hline$"$ & (M) Frosted glass vs. River stone & 67 & 55 \\
\hline ", & (M) Smooth glass vs. River stone & 67 & 68 \\
\hline ", & (C) Vermont slate vs. River stone & 66 & 56 \\
\hline$"$ & (M) Acrylic vs. River stone & 60 & 55 \\
\hline
\end{tabular}


during this time. There was not as high a similarity between monthly artificial substrates and river stones and values for all 7 comparisons were between $60 \%$ and $78 \%$. Cumulative plates did not seem much, if any, more similar to river stones than did monthly plates.

The coefficient of community, percentage of total genera present on both substrates of pairs being compared, was from $26 \%$ to $63 \%$ when monthly Vermont slate replicates were compared (Table 4 ). The coefficient of community was much higher, $63 \%$ to $80 \%$, when combined May through December data were compared for each of the substrates (Table 4). The July data for each of the artificial substrates compared with river stone communities yielded coefficients of community of 45 to 68 (Table 4).

\section{Discussion}

In most instances the three most abundant genera on various kinds of artificial substrates and on river stones were the same, although their relative position in the top three often varied. It seems that any of several artificial substrates could provide a reasonably good idea of the dominant algal genera and species present on river stones.

The number of genera on artificial substrates (sampled monthly) tended to be higher than the number on river stones. This phenomenon has also been reported to occur in other streams by Patrick et al. (1954).

The use of artificial substrates in periphyton studies provides soms advantages to the investigator, but also includes several hazards. Two of these hazards merit a brief discussion here. It is clear that the length of time required to colonize a substrate varied a great deal with season. If the object of future studies is to allow the substrate to become colonized as heavily as the natural substrate or to establish a 'constant standing crop' as described by Elwood \& Nelson (1972), then a 2-wk colonization period, like that recommended by Weber \& Raschke (I970) and by others might suffice in summer. But, in winter even a month would be too little colonization time. Elwood \& Nelson (1972) found that at least six weeks were required to attain a 'constant standing crop' on natural substrates in an artificial stream.

Colonization of subtrates placed near the river surface, like Weber \& Raschke's ( 1970) might colonize faster than those near the bottom, where they were placed in this study. Placement of artificial substrates near the river bottom, in the same environment as the natural sub- strate, enhances the ecological value of the data collected on them (Brown \& Austin, 1971).

This study also revealed that short term (monthly) colonization rates on clean plates are sometimes poor indicators of changes or trends in the development of the periphyton community on natural substrates. For example, in June, when colonization rates were high, algae on natural substrates became much less abundant. In November, when colonization rates declined on monthly plates, algal density on natural substrates increased markedly. In December, 1974 and January, 1975, when almost no colonization occurred, algal density on natural substrates and cumulative Vermont slate increased. Thus, it seems that if the objective of future studies is to detect moderate or large changes in the existing periphyton community that result from environmental modification, samples should be collected from river stones or from artificial substrates that have been submerged for several months. Obviously, it would be unrealistic to attempt to leave artificial substrates near the river surface for an extended period of time, for if they were not swept away by ice or floating debris, they would likely be destroyed by vandals. It would, however, be realistic to leave artificial substrates submerged on the river bottom if they were placed on 'detritus-free' holders like those described in Fig. I. Plates placed in the Susquehanna River in January, 1975 have remained in place for over 29 months. The plates did not seem to have been affected by a major flood in September, 1974. If a single type of artificial substrate is to be used, frosted acrylic would be a good choice for it: (I) is readily available; (2) has a uniform surface texture; (3) is easily sawed, drilled and glued, and (4) colonizes well with algae that seem as representative of the algal community on river stones as the communities that developed on other artificial substrates. Although all of the artificial substrates in this study faced the current at about $5^{\circ}$ from horizontal, plates could also be placed at other angles from the bottom.

It is not at all certain that the use of artificial substrates in this study was successful in reducing sample variability to an appreciable extent. There was usually substantial variability in numbers of algal units in replicated samples taken from Vermont slates, in spite of their homogenous surfaces. Sometimes there differences were as large or larger than differences found in samples from river stones. 


\section{Acknowledgements}

We thank Douglas Thompson for collecting the samples and Andrew Gurzynski for his help in sample processing.

\section{Summary}

The Susquehanna River, Pennsylvania, U.S.A. was chosen as a site to study periphyton colonization on a river bottom. Artificial substrates of smooth glass, frosted glass, Vermont slate, sandy slate, and frosted acrylic were fastened to detritus free periphyton samplers and placed on the river bottom. Exposed substrates were collected monthly by scuba divers employing bar-clamp samplers. Some substrates were left on the river bottom for several months to collect cumulative periphyton samples. Natural river stones were also collected for comparison.

Diatoms were the most abundant algae on the substrates. The genera Nitzschia, Navicula, Cocconeis, Synedra, Asterionella, Gomphonema, Meridion, Cymbella, Fragilaria, Stephanodiscus, Cyclotella, Diatoma, and Melosira were all well respresented. Substrates with one month exposure times displayed very little periphyton colonization from December through April. From May through November, one month exposure periods resulted in periphyton communities with densities varying from less than $\mathrm{I}, 000 \mathrm{units} / \mathrm{mm}^{2}$ to around I0,000 units $/ \mathrm{mm}^{2}$. October samples accumulated the densest communities.

Cumulative substrates generally maintained the densest periphyton communities in the winter months with density decreasing from June through October. Natural river stones yielded densities of periphyton most similar to cummulative substrates. The greatest periphyton density found was on a river stone in February $\left(2 \mathrm{I}, 850\right.$ units $\left./ \mathrm{mm}^{2}\right)$.

In most cases, the various substrates used had the same dominant genera and any of the substrates could provide a satisfactory estimate of dominant genera in the periphyton. In using benthic substrates one must be aware, however, that natural river stones are collecting cumulative algal samples and in most instances one month exposure periods of artificial substrates are not adequate. Frosted acrylic appears to be a good candidate for continued use as an artificial substrate because of it's ready availability, uniform surface and ease of manipulation.

\section{References}

Brown, S. \& Austin, A. 1971. A method of collecting periphyton in lentic habitats with procedures for subsequent sample preparation and quantitative assessment. Int. Revue ges. Hydrobiol. 56: 557-580.

Butcher, R. W. 1947. Studies in the ecology of rivers. IV. The algae of organically enriched water. J. Ecol. 35: 186-I9I.

Dam, H. van. 1974. The suitability of diatoms for biological water assessment. Hydrobiological Bull. 8 (3): 274-284.

Elwood, J. \& Nelson, D. 1972. Periphyton production and grazing rates in a stream measured with a ${ }^{32} \mathrm{P}$ material balance method. OIKOS 23: 295-303.

Gale, W. 1975. Ultrasonic removal of epilithic algae in a barclamp sampler. J. Phycol. I I: 472-473.

Gale, W. \& Lowe, R. 1971. Phytoplankton ingestion by the fingernail clam, Sphaerium transversum (Say), in Pool 19, Mississippi River. Ecol. 52: 507-513.

Gale, W. \& Thompson, J. 1974. Aids to benthic sampling by scuba divers in rivers. Limnol. and Oceanogr. 19: 1004-I007.

Hohn, M. H. \& Hellerman, J. 1963. The taxonomy and structure of diatom populations from three Eastern North American Rivers using three sampling methods. Amer. Microscop. Soc. Trans. 82: 250-329.

Lowe, R. L. 1972. Diatom population dynamics in a central lowa drainage ditch. Iowa State J. Res. 4I ( I): 7-59.

Lowe, R. L. \& McCullough, J. M. I974. The effect of sewagetreatment-plant effluent on diatom communities in the North Branch of the Portage River, Wood County, Ohio. Ohio J. Sci., 74 (3): I54-16 I.

Patrick, R. I968. The structure of diatom communities in similar ecological conditions. Amer. Mid. Natur. I02 (924): I73I83.

Patrick, R. 1973. Use of algae, especially diatoms, in the assessment of water quality. In Biological Methods for the Assessment of Water Quality, ASTM STP 528, American Society for Testing and Materials, pp. 76-95.

Patrick, R. \& Hohn, M. H. 1956. The diatometer-a method for indicating the conditions of aquatic life. Proc. Am. Petrol. Inst., Sec. 3, 36: 332-338.

Patrick, R., Hohn, M. \& Wallace, J. 1954. A new method for determining the patterns of the diatom flora. Notulae Naturae, Acad. Natural Sci., Phila. 259: I-I 2.

Van Landingham, S. I976. Comparative evaluation of water quality on the St. Joseph River (Michigan and Indiana, U.S.A.). By three methods of algal analysis. Hydrobiol. 48 (2):

Weber, C. \& Raschke, R. 1970. Use of a floating periphyton sampler for water pollution surveillance. Fed. Wat. Pol. Contr. ....Admin. Reprint. 22 pp.

Whittaker, R. \& Fairbanks, C. 1958. A study of the plankton copepod communities in Columbia Basin, southeastern Washington. Ecol., 39: 46-65. 